\title{
SOME ASPECTS OF SATELLITE IMAGERY INTEGRATION FROM EROS B AND LANDSAT 8
}

\author{
A. Fryskowska ${ }^{a}$, M. Wojtkowska ${ }^{\text {a, }}$ P. Delis ${ }^{\text {a, }}$, A. Grochala ${ }^{\text {a }}$ \\ Department of Remote Sensing and Photogrammetry, Geodesy Institute, Faculty of Civil Engineering and Geodesy, \\ Military University of Technology, Warsaw, Poland - (anna.fryskowska, michalina.wojtkowska, paulina.delis, \\ aleksandra.grochala)@wat.edu.pl;
}

\section{Commission VII, WG VII/6}

KEY WORDS: Image Processing, Fusion data, Pan-sharpening, Multispectral imagery, IHS resolution merge, Brovey transform

\begin{abstract}
:
The Landsat 8 satellite which was launched in 2013 is a next generation of the Landsat remote sensing satellites series. It is equipped with two new sensors: the Operational Land Imager (OLI) and the Thermal Infrared Sensor (TIRS). What distinguishes this satellite from the previous is four new bands (coastal aerosol, cirrus and two thermal infrared TIRS bands). Similar to its antecedent, Landsat 8 records electromagnetic radiation in a panchromatic band at a range of $0,5-0,9 \mu \mathrm{m}$ with a spatial resolution equal to $15 \mathrm{~m}$. In the paper, multispectral imagery integration capabilities of Landsat 8 with data from the new high resolution panchromatic EROS B satellite are analyzed. The range of panchromatic band for EROS B is $0,4-0,9 \mu \mathrm{m}$ and spatial resolution is $0.7 \mathrm{~m}$. Research relied on improving the spatial resolution of natural color band combinations (bands: 4,3,2) and of desired false color band composition of Landsat 8 satellite imagery. For this purpose, six algorithms have been tested: Brovey's, Mulitplicative, PCA, IHS, Ehler's, HPF. On the basis of the visual assessment, it was concluded that the best results of multispectral and panchromatic image integration, regardless land cover, are obtained for the multiplicative method. These conclusions were confirmed by statistical analysis using correlation coefficient, ERGAS and R-RMSE indicators.
\end{abstract}

\section{INTRODUCTION}

In recent years, intensive development of high resolution satellite techniques is noticed. A new generation of satellites allow recording images with a pixel size less than $1 \mathrm{~m}$. They offers panchromatic data with high spatial resolution, but characterized by relatively low potential interpretation. Coloured images with enhanced spatial resolution (ie. sharpened images) can be used to support geographic information systems (Mori, 2002), creation of land cover maps (Ochi, 2008), monitoring changes in ecosystems (Haruyama et al, 2010) and forest monitoring (cultivation planning and management, monitoring the health of trees) (Kosaka et al, 2005).

The Landsat 8 satellite which was launched in 2013 is a next generation of the Landsat remote sensing satellites series. It is equipped with two new sensors: the Operational Land Imager (OLI) and the Thermal Infrared Sensor (TIRS). What distinguishes this satellite from the previous is four new bands (coastal aerosol, cirrus and two thermal infrared TIRS bands). The Landsat 8 mission objective is to provide timely, high quality visible and infrared images of all landmass and nearcoastal areas on the Earth, continually refreshing an existing Landsat database. It offers 16-day repetitive Earth coverage, an 8-day repeat with a Landsat 7 offset. Landsat 8 records electromagnetic radiation in a panchromatic band at a range of $0,5-0,9 \mu \mathrm{m}$ with a spatial resolution equal to $15 \mathrm{~m}$.

EROS B satellite was launched into orbit on April 25, 2006. This high resolution satellite orbits the Earth at a height between 516 and 524 kilometers, collecting black and white imagery with an average resolution of 70 centimeters. This is the only high resolution satellite that can be tasked to collect nighttime imagery. This data has a wide array of applications including urban sprawl measurements, light pollution detection and population estimates.

It is assumed that a pan-sharpening can be performed for images for which the spatial resolution ratio does not exceed 4 . For Landsat 8 and EROS B this ratio is very high and equals 18 . However, the aim of this study is improving the spatial resolution of natural color band combinations (bands: 4,3,2) of Landsat 8 satellite imagery based on data from the satellite Eros B. The combination of the multispectral imagery with the high spatial resolution panchromatic imagery, could be a valuable source of data to perform various remote sensing analysis to better monitor and understand processes occurring in the environment.

\section{FUSION DATA METHODS}

The main problem with integration image data with different spatial and spectral resolution is to preserve both spectral and spatial component. There are a lot of algorithms of the multispectral and panchromatic imagery integration. These algorithms vary in action and not all of them allow to achieve positive results of the pan-sharpening.

The most commonly pan-sharpening methods are: RGB to IHS, Brovey's multiplicative methods, Principal Components Analysis, Ehler fusion, fusion based on the high-pass filter HPF (high-pass filter). Each of these methods uses a different image properties. One of the most popular method is IHS algorithm. This algorithm requires relatively little computing power. It uses three bands (R, G, B) and converts them into color space IHS. In the IHS color space a component of intensity $I$ is changed with a band of the panchromatic image.

\footnotetext{
* Corresponding author
} 
Unfortunately, this method causes colour distortion (GonzalezAudicana, 2004).

Multiplicative transform relies on multiplication pixel value panchromatic image with every pixel of bands multispectral image. This algorithm in terms of the calculation is relatively simple (Helmy, 2010).

Brovey's method preserves the spectral properties of the multispectral image pixels but changes the overall brightness of the panchromatic image. One of the disadavantage is that it works only for three spectral bands (Vijayaraj, 2004).

Principal Components Analysis method uses arbitrary number of spectral bands of the multispectral image. The method converts the imaging ranges correlated with a new set of unrelated image (component). Thus, the pixels of the resulting image will contain the new coordinates, and also the largest information in this area for which the histogram shows the greatest divergence. In the result, a new color image is generated. It is characterized by very well-preserved colour balance of the multispectral image and the high spatial resolution of the panchromatic image (Chavez, et al, 1991).

Ehler's fusion is a method which is similar to the IHS algorithm. However, in the opposition to the standard algorithm which change RGB to IHS model space, Ehler's fusion allow for selection any number of bands of the multispectral image. In this case, the algorithm realizes several times the conversion of the colour model space until using all bands. Then, a transformation of the panchromatic image with a intenstity component to the frequency domain (using Fast Fourier Transform) is performed. In order to extract the details, it is possible to select the appropriate high-pass filter for the panchromatic image and a low pass filter for the multispectral image (Ehler, 2004).

A basis of the HPF method is the high pass filtration. A size of the filter's matrix is dependent on a ratio between the spatial resolution of the panchromatic and the multispectral images. This ratio is multiplied two times. The value of the center pixel matrix is its weight. Higher value means the weight of the matrix increases the visual characteristics of the image. It has been done by reducing false edges of objects and increase its value colour saturation, which leads to increase the contrast of the resulting image.

The HPF method includes three steps: high-pass filtering of the panchromatic image, addition each of the multispectral image bands to the filtered image and a histogram matching of the resulting image with the corresponding bands of the multispectral image. The HPF method improves colour reproduction on the final image while preserving detail of objects of the panchromatic image.

\section{METHODS OF PAN-SHARPENING RESULTS ASSESSMENT}

The most common methods of evaluation of pansharpening methods are visual and statistical assessment based on mathematical indicators (Kędzierski et al., 2014). The visual assessment includes such aspects as a preservation of image sharpness, details and colour fidelity in image being the result of the multispectral and panchromatic image integration.
The indicators of quality assessment of pan-sharpened image are correlation coefficient, R-RMSE and ERGAS, (Helmy et al., 2010), (Straight et al, 2008).

First of indicators, correlation coefficient is a measure of similarity between the pan-sharpened and the multispectral images.

$$
\begin{gathered}
\rho=\frac{\sum_{i, j}\left[g_{1}\left(c_{1}, r_{1}\right)-\overline{g_{1}}\right]\left[g_{2}\left(c_{2}, r_{2}\right)-\overline{g_{2}}\right]}{\sqrt{\sum_{i, j}\left[g_{1}\left(c_{1}, r_{1}\right)-\overline{g_{1}}\right]^{2} \sum_{i, j}\left[g_{2}\left(c_{2}, r_{2}\right)-\overline{g_{2}}\right]^{2}}} \\
\overline{g_{1}}=\frac{1}{n} \sum_{i, j} g_{1}\left(c_{1}, r_{1}\right) \quad \overline{g_{2}}=\frac{1}{n} \sum_{i, j} g_{2}\left(c_{2}, r_{2}\right)
\end{gathered}
$$

where:

$\mathrm{g}$ - pixel value

$\mathrm{c}_{1}, \mathrm{r}_{1}$ - pixel coordinates of the pan-sharpened image

$c_{2}, r_{2}$ - pixel coordinates of the multispectral image

$\mathrm{n}$ - number of pixels

$\mathrm{i}, \mathrm{j}$ - number of column and row in a correlation window

The next indicator of quality assessment of pan-sharpened image is the relative mean error R-RMSE. This indicator compares the DN values of pixels of the output image with DN values of pixels in the multispectral image (Kroll i Stedinger, 1996).

$$
R-R M S E_{k}=\sqrt{\frac{\sum_{i=1}^{N}\left(\frac{D N_{\text {fused }(k) i}-D N_{\text {orygina }(k) i}}{\left.D N_{\text {orygina }(k)}\right)^{2}}\right.}{N}}
$$

where $\mathrm{DN}$ - the value of the i-th pixel in the k-band.

ERGAS (Relative Dimension Less Global Error in Synthesis) compares pixel value of the multispectral and the pan-sharpened image. It takes into account the difference of the spatial resolution and wavelength recorded in an analyzed area. The ideal value for ERGAS is zero. ERGAS indicator equals zero means that there is a lack of losing the quality of the optical density between pixels of the pan-sharpened and the multispectral images. It is assumed that the value ERGAS less than 3 indicates a satisfactory quality of pan-sharpened image (Wald, 2002).

$$
E R G A S=100 \cdot \frac{h}{l} \sqrt{\frac{1}{K} \sum_{k=1}^{K}\left(\frac{R M S E(k)}{\mu_{0}}\right)^{2}}
$$

where:

$\mathrm{h}-\mathrm{GSD}$ of the panchromatic image

$1-$ GSD of the multispectral image

$\mathrm{K}$ - number of multispestral image bands

\section{DATA PROCESSING}

In order to test the possibility of improving the spatial resolution of Landsat 8 multispestral imagery based on panchromatic image acquired by Eros B satellite, above data fusion algorithms were tested. The algorithms have been tested for different types of land cover. 


\subsection{Data description}

Used in the research image data present an area of Gdansk (Poland). The operation of the algorithms were tested on three fragments of a satellite scene. These fragments present different types of land cover. The analyzed area includes a high urban area with flowing through the city river (Figure 1), as well as the wooded hills (Figure 2) and the open area included irrigation fields (Figure 3).

The multispectral imagery from Landsat 8 system were acquired in February 2015 and panchromatic EROS B imagery was collected five years earlier. Both images have been processed to the same reference system (UTM WGS-84 zone 33).
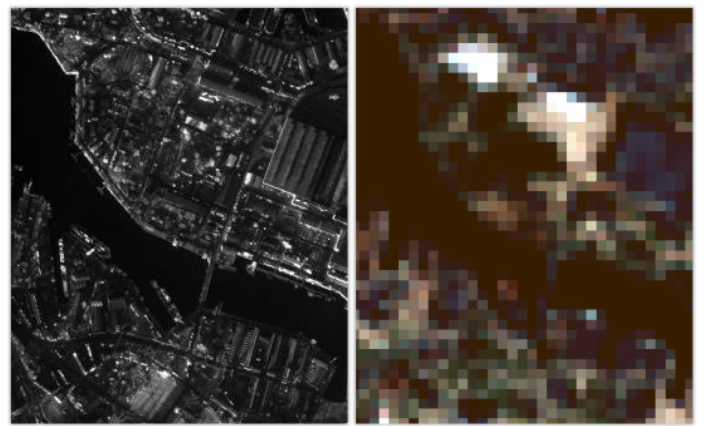

Figure 1. High urban area. Left image: panchromatic imagery from Eros. Right image: multispectral imagery from Landsat- 8 in the band combination 4 (red), 3 (green), 2 (blue)
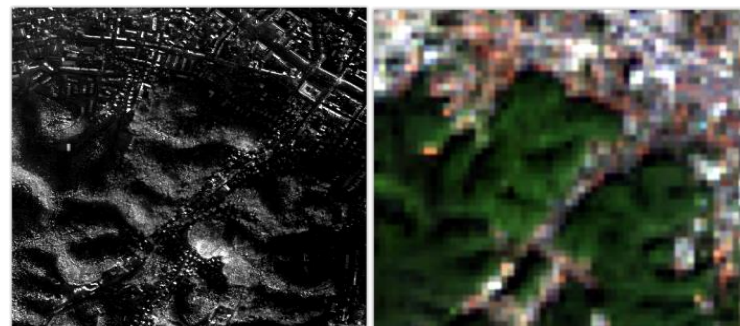

Figure 2. Forest area. Left image: panchromatic imagery from Eros. Right image: multispectral imagery from Landsat- 8 in the band combination 4 (red), 3 (green), 2 (blue)
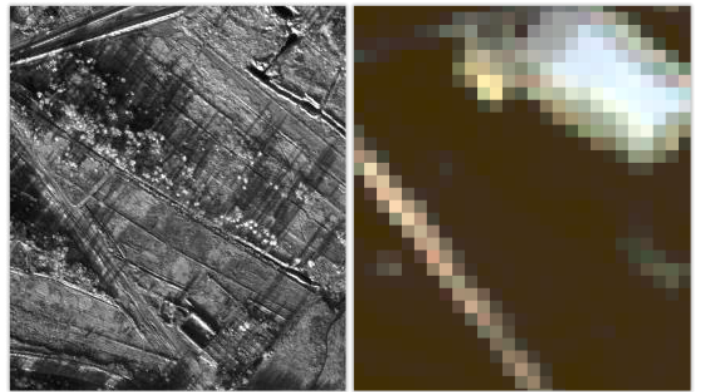

Figure 3. Open area. Left image: panchromatic imagery from Eros. Right image: multispectral imagery from Landsat- 8 in the band combination 4 (red), 3 (green), 2 (blue)

A characteristic feature of the test data is very high ratio between spatial resolution of the two imagery from Landsat and EROS B. What is more, the images from both satellites are difficult in the interpretation.

\subsection{Results and discussion}

The following figures present results of the integration of the multispectral image data from Landsat 8 with the panchromatic image data from Eros B. In order to data processing the software ERDAS IMAGINE 2013 were used.
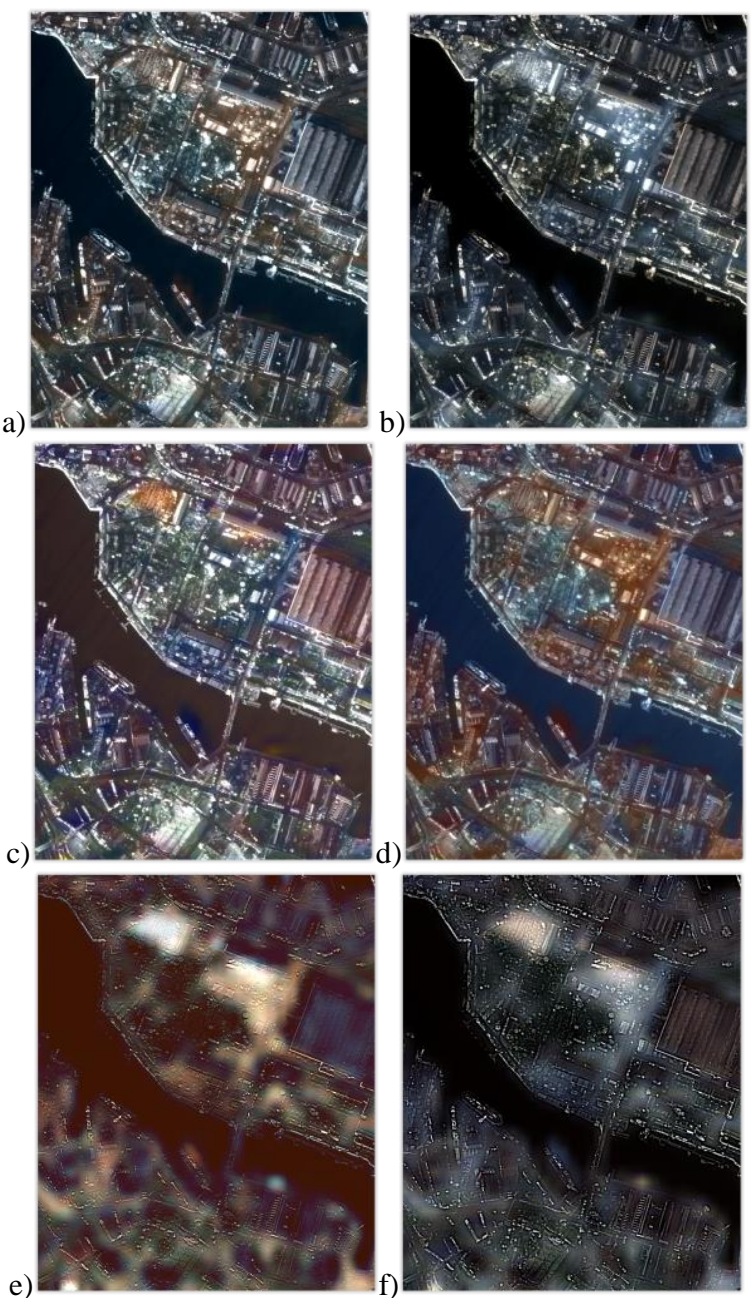

Figure 4. Results of pan-sharpening of the urban area image based on: Brovey's (a), Multiplicative (b), PCA (c), IHS (d), Ehler's (e), HPF (f) methods

Based on visual assessment of above figures it can be concluded that for the urban area the best results were obtained using the multiplicative fusion method. Relatively good results have given with the Brovey's and PCA methods. The image sharpened with IHS method IHS is characterized by colour distortion. The worst results were obtained with the Ehler's and HPF methods. In these cases the effect of high-pass filtering can be seen. For all images, regardless of the data fusion method, the river has been very well mapped. 


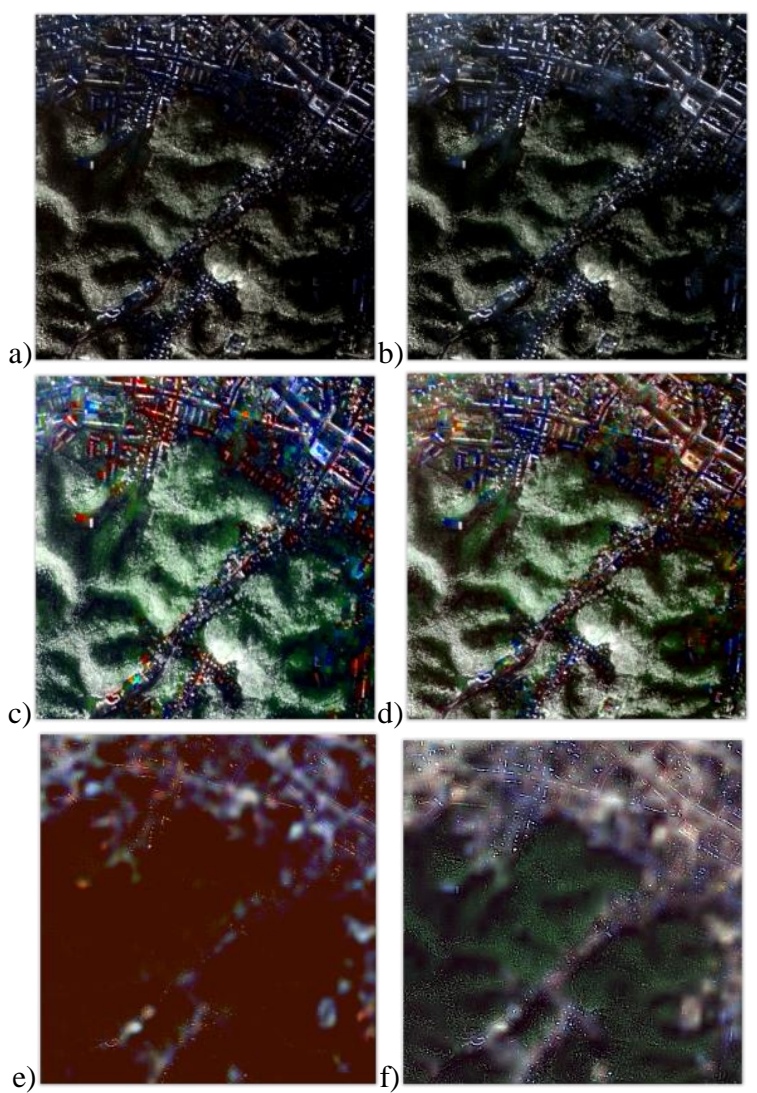

Figure 5. Results of pan-sharpening of the forest area image based on: Brovey's (a), Multiplicative (b), PCA (c), IHS (d),

Ehler's (e), HPF (f) methods

For the wooded hills, the best results were obtained with the Brovey's and multiplicative methods. In cases the PCA and IHS method, the biggest colour distortion have been noted for the low buildings, which are situated in the north-eastern part of figures. Similar to the high urban area, the worst results were obtained for Ehler's and HPF methods. Despite increasing the spatial resolution, pan-sharpened images appear as blur, with a low resolution. In addition, the pan-sharpened image generated with the Ehler's fusion is characterized by colour distortion.
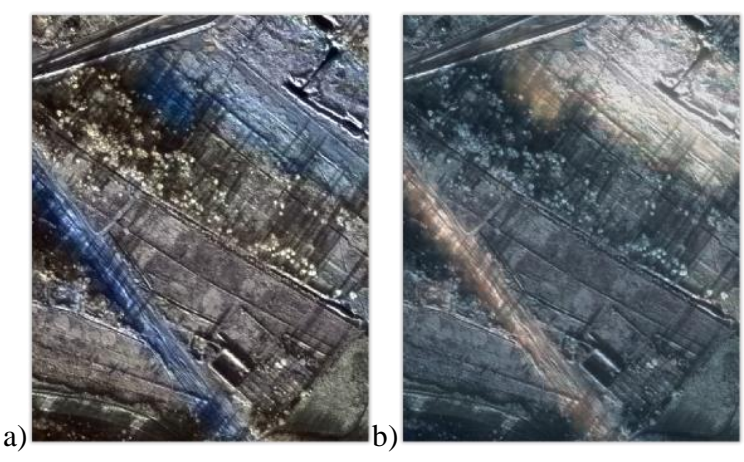
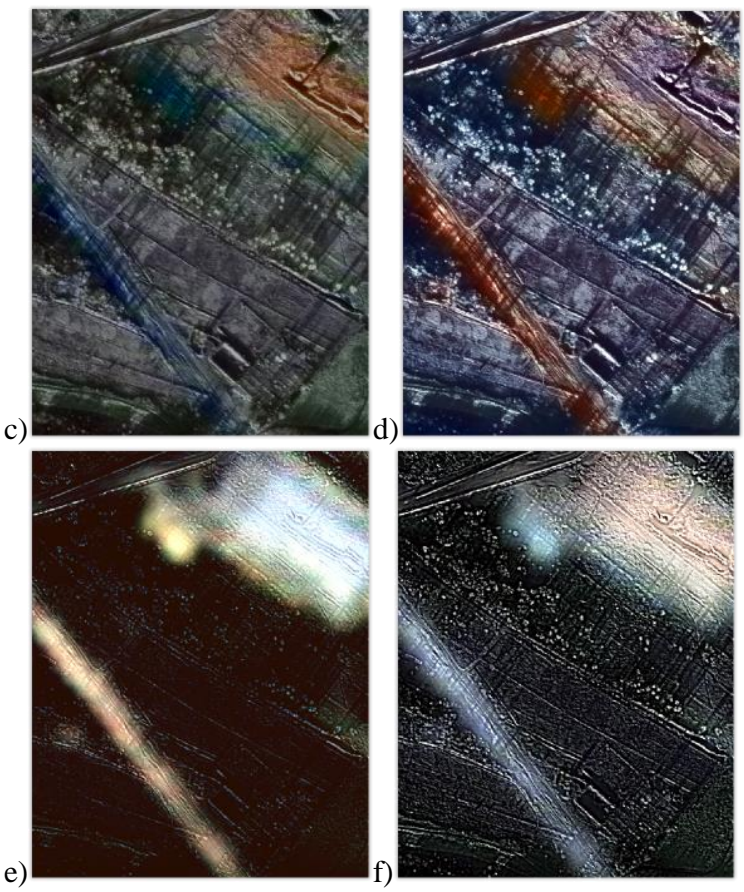

Figure 6. Results of pan-sharpening of the open area image based on: Brovey's (a), Multiplicative (b), PCA (c), IHS (d), Ehler's (e), HPF (f) methods

Apart from data fusion method, none of the pan-sharpened images of open area is satisfactory. On each image, a colour distortion is a visible, especially for the road. Due to the fact that the multispectral imagery acquired by Landsat 8 was made five years later than the Eros B imagery, some changes in the land cover are noticeable. One of them is an industrial hall which is visible in the north-eastern part of the Figures 6.

The results of the visual assessment have been verified using statistical indicators. In this order, correlation coefficient, relative mean error between pixel density and sharpened image multispectral (R-RMSE) and ERGAS were used. Above indicators of quality have been designated in the Modeler tool of ERDAS IMAGINE 2013 software.

\begin{tabular}{|ccccc|}
\hline \multicolumn{5}{|c|}{ corelation coefficient } \\
\hline & open area & urban area & river & forest \\
\hline Brovey & 0,11 & 0,27 & 0,68 & 0,07 \\
Multi. & 0,52 & 0,5 & 0,04 & 0,22 \\
PCA & 0,1 & 0,27 & 0,04 & 0,05 \\
IHS & 0,02 & 0,32 & 0,05 & 0,05 \\
Ehler & 0,88 & 0,73 & 0,62 & 0,79 \\
HPF & 0,51 & 0,53 & 0,02 & 0,46 \\
\hline
\end{tabular}

Table 1. Correlation coefficients of the pan-sharpened images

Values of correlation coefficient determined for pan-sharpened images derived from one satellite, for example World View-2 are order of 0.8-0.9 (Samadzadegan and DadrasJavan, 2011). Thus values obtained in this studies should be regarded as low. The reason is the fact that spatial resolution of the multispectral image is the correlation related to images for which image resolution multispectral is multiple lower than the panchromatic image. What is more, the low correlation coefficient could be also affected by large (5-year) interval between imageries. 
Taking into consideration land cover, the highest correlation results were obtained for the urban area, and the lowest for river. The low correlation coefficients for river is due to uniform texture and lack of detail in the river. The highest value of the correlation coefficient between the pan-sharpened image and multispectral image obtained with the Ehler's algorithm. The relatively high value of the correlation coefficient was also observed for pan-sharpened image using HPF method. Paradoxically, both methods were considered to be the worst during visual assessment. The reason for this is that pansharpened images which were obtained with these methods are the most similar to low resolution multispectral images. Multiplicative method considered to be the best in visual analysis is characterized by relatively high correlation coefficient.

\begin{tabular}{|ccccc|}
\hline \multicolumn{5}{|c|}{ R-RMSE } \\
\hline & open area & urban area & river & forest \\
\hline Brovey & 1,0 & 1,0 & 1,0 & 1,0 \\
Multi. & 284 & 256 & 172 & 257 \\
PCA & 0,3 & 0,2 & 0,3 & 0,2 \\
IHS & 0,2 & 0,1 & 0,1 & 0,1 \\
Ehler & 0,1 & 0,1 & 0,1 & 0,1 \\
HPF & 0,2 & 0,2 & 0,1 & 0,1 \\
\hline
\end{tabular}

Table 2. R-RMSE indicators of the pan-sharpened images

\begin{tabular}{|ccccc|}
\hline \multicolumn{5}{c|}{ ERGAS } \\
\hline & open area & urban area & river & forest \\
\hline Brovey & 58 & 57 & 57 & 57 \\
Multi. & 416 & 125 & 960 & 176 \\
PCA & 17 & 17 & 19 & 9 \\
IHS & 11 & 12 & 6 & 6 \\
Ehler & 5 & 6 & 5 & 5 \\
HPF & 9 & 14 & 6 & 6 \\
\hline
\end{tabular}

Table 3. ERGAS indicators of the pan-sharpened images

Both for the R-RMSE and ERGAS indicators lower values mean better results of data fusion. What is interested, for the Mulitplicative method, the highest values of coefficients were obtained. Regardless of the land cover type, values of R-RMSE and ERGAS coefficients are similar. What is more, the lowest errors were obtained for the images which were pan-sharpened with the Ehler's and HPF methods.

\section{SUMMARY AND CONCLUSIONS}

Based on the research, it have been proved that improving the spatial resolution of natural color band combinations (bands: $4,3,2$ ) of Landsat 8 satellite imagery based on data from the satellite Eros B allows to obtain positive results. For this purpose, six algorithms have been tested: Brovey's, Mulitplicative, PCA, IHS, Ehler's, HPF. On the basis of the visual assessment, it was concluded that the best results of multispectral and panchromatic image integration, regardless land cover, are obtained for the multiplicative and for Brovey's method. These conclusions were confirmed by statistical analysis using correlation coefficient, ERGAS and R-RMSE indicators. The visual assessment reveals that the worst results were obtained for Ehler's and HPF methods. On the other hand, these methods are characterized by best results of the statistical analysis. The combination of Landsat 8 multispectral imagery with the high spatial resolution Eros B panchromatic imagery, could be a valuable source of data to perform various remote sensing analyzes.

\section{ACKNOWLEDGEMENTS}

This paper has been supported by the Military University of Technology, the Faculty of Civil Engineering and Geodesy, Department of Remote Sensing and Photogrammetry as a research project 933/2016.

\section{REFERENCES}

Chavez, P. S., Sides, S., Anderson, J., 1991. Comparison of three different methods to merge multiresolution and multispectral data: Landsat TM and SPOT panchromatic, Photogrammetric Engineering \& Remote Sensing 57(3), pp. 295-303.

Ehlers, M. 2004. Spectral Characteristics Preserving Image Fusion Based on Fourier Domain Filtering, In: Remote Sensing for Environmental Monitoring, GIS Applications, and Geology IV, Proceedings of SPIE, pp. 1-13.

González-Audícana, M., Otazu, X., 2004. Comparison between Mallat's and the a'trous discrete wavelet transform based algorithms for the fusion of multispectral and panchromatic images, International Journal of Remote Sensing 26(3), pp. 595-614.

Haruyama, Y., Ono, M., Tomiyama, N., 2010. Monitoring changes in ecosystems in Latin America by ALOS, International Archives of the Photogrammetry, Remote Sensing and Spatial Information Services, Vol. XXXVIII, Part 8. Kyoto, Japan 2010.

Helmy, A. K., Nasr, A. H., El-Taweel, Gh. S.,2010. Assessment and Evaluation of Different Data Fusion Techniques, International Journal of Computers 4(4), pp. 107-115.

Kedzierski M., Wilinska M., Wierzbicki D., Fryskowska A., Delis P., 2014. Image data fusion for flood plain mapping, 9th International Conference Environmental Engineering (9th ICEE), Vilnius, Lithuania, May 22-23, 2014.

Klonus, S., 2008. Comparison of pan-sharpening algorithms for combining Radar and multispectral data), Archives of the Photogrammetry, Remote Sensing and Spatial Information Services, Vol. XXXVII, Part B6b, Beijing, 2008.

Kosaka, N., Akiyama, T., Tsai, B., Kojima, T., 2005. Forest type classification using data fusion of multispectral and panchromatic high-resolution satellite Imageries, IEEE International Geoscience and Remote Sensing Symposium, Vols 1-8, Proceedings Book Series: IEEE International Symposium on Geoscience and Remote Sensing (IGARSS), pp. 2980-2983.

Kroll, C., Stedinger, J., 1996. Estimation of moments and quantiles using censored data, Water Resources Research 32(4), pp. 1005-1012. 
Mori, M., Application of IKONOS imagery on geographic information systems (2002),In: IEEE International Symposium on Geoscience and Remote Sensing (IGARSS), pp. 3352-3354.

Ochi, S., 2000. Land use characterization using landcover objects from high resolution satellite image, Archives of the Photogrammetry, Remote Sensing and Spatial Information Services, Vol. XXXVII, Part B7. Beijing, 2008.

Samadzadegan, F., DadrasJavan F. 2010. Evaluating the potential of image quality metrics for quality assessment of high resolution pan-sharpen satellite imagery in urban area, The 2010 Canadian Geomatics Conference and Symposium of Commission, ISPRS Convergence in Geomatics - Shaping Canada's Competitive Landscap, Archives of the Photogrammetry, Remote Sensing and Spatial Information Services, Vol. XXXVIII - Part 1.

Strait, M., Rahmani, S., Merkurev, D., 2008. Evaluation of pansharpening methods, UCLA, Los Angeles, CA, Tech. Rep.

Vijayaraj, V. O'Hara, C. G., Younan, N. H., 2004. Quality analysis of pansharpened images, In: Proceedings of IEEE International Geoscience and Remote Sensing Symposium, vol. 1, pp: 85-88.

Wald, L. 2002. Data Fusion: Definitions and Architectures Fusion of Images of Different Spatial Resolutions, Les Presses, Ecole des Mines de Paris, Paris, France. 